\title{
Aging characteristics of blue InGaN micro-light emitting diodes at an extremely high current density of $3.5 \mathrm{kA} / \mathrm{cm}^{2}$
}

\author{
Pengfei Tian ${ }^{1,2}$, Ahmad Althumali ${ }^{2}$, Erdan $\mathrm{Gu}^{2}$, Ian M. \\ Watson $^{2}$, Martin D. Dawson ${ }^{2}$ and Ran Liu ${ }^{1}$ \\ ${ }^{1}$ Institute for Electric Light Sources, Fudan University, Engineering Research Center \\ of Advanced Lighting Technology, Ministry of Education, Shanghai, China \\ ${ }^{2}$ Institute of Photonics, Department of Physics, University of Strathclyde, Glasgow, \\ United Kingdom \\ E-mail: pftian@fudan.edu.cn and erdan.gu@strath.ac.uk
}

February 2016

\begin{abstract}
The aging characteristics of blue InGaN micro-light emitting diodes (micro-LEDs) with different sizes have been studied at an extremely high current density $3.5 \mathrm{kA} / \mathrm{cm}^{2}$ for emerging micro-LED applications including visible light communication, micro-LED pumped organic lasers and optogenetics. The light output power of micro-LEDs first increases and then decreases due to the competition of $\mathrm{Mg}$ activation in $p$-GaN layer and defect generation in the active region. The smaller micro-LEDs show less light output power degradation compared with larger microLEDs, which is attributed to the lower junction temperature of smaller micro-LEDs. It is found that the high current density without additional junction temperature cannot induce significant micro-LED degradation at room temperature but the combination of the high current density and high junction temperature leads to strong degradation. Furthermore, the cluster LEDs, composed of a micro-LED array, have been developed with both high light output power and less light output degradation for micro-LED applications in solid state lighting and visible light communication.
\end{abstract}

Keywords: Micro-light emitting diodes, aging, InGaN, high current density, defect

\section{Introduction}

Micro-light emitting diodes (micro-LEDs) have demonstrated applications including visible light communication (VLC) [1, 2], micro-LED pumped organic lasers [3, 4], optogenetics [5], micro-display [6], direct LED writing [7], and solid-state lighting (SSL) [8], due to their special characteristics of small size, localized light emission, and high heat dissipation ability $[9,10]$. In comparison with conventionally used broad-area LED devices with an operation current density of around $100 \mathrm{~A} / \mathrm{cm}^{2}[11,12,13]$, micro-LEDs can operate at extremely high injection current densities of several $\mathrm{kA} / \mathrm{cm}^{2}$ to achieve high density of light output power and high modulation bandwidth in their applications. 
Specifically, a very high modulation bandwidth of up to $\sim 435 \mathrm{MHz}$ has been achieved using a $44 \mu \mathrm{m}$ micro-LED at an injection current density of $\sim 4.6 \mathrm{kA} / \mathrm{cm}^{2}$ for VLC [1]; a $30 \mu \mathrm{m}$ micro-LED can sustain an injection current density of $10 \mathrm{kA} / \mathrm{cm}^{2}$ to achieve a high density of light output power for organic laser pumping [3]. The micro-LED characteristics at high current densities, including electrical characteristics, light output, electroluminescence spectral shift, efficiency droop and optical modulation bandwidth, have been widely studied $[1,9,10,14]$, but the aging and reliability characteristics at such high current densities, key properties of how the micro-LED performance changes with increasing driving time, have not been investigated.

The lifetime of conventional broad-area LEDs is defined based on the time of $50 \%$ light output degradation for the display industry or $70 \%$ light output degradation for the lighting industry with a lifetime of tens of thousands of hours [15]. Similarly, we expect that different lifetime standard should be defined in each application of microLEDs, due to the different driving current densities and LED sizes. On the one hand, extremely high current density and light output density are required for applications in VLC and organic laser pumping [1,3], and relatively low current densities are used for micro-displays [6]. On the other hand, the micro-LED size has strong effect on the LED lifetime, as discussed in next paragraph. In this study we measured the light output powers of micro-LEDs with different sizes until they reach 90\% (or less) light output degradation (L90). These measurements were carried out at an extremely high current density of $3.5 \mathrm{kA} / \mathrm{cm}^{2}$. Such a high current density can greatly accelerate the aging test of these micro-LEDs. Note that the L90 lifetime is useful to compare the aging behavior of micro-LEDs with different sizes in this study, but also can be used to predict the micro-LED reliability.

The extremely high injection current density and greatly reduced self-heating effect of micro-LEDs may lead to different aging behaviors from broad-area LEDs $[9,10]$. It has been reported, at an operation current density around $100 \mathrm{~A} / \mathrm{cm}^{2}$, broad-area LEDs suffered from current-induced stress by injected electrons and thermal-induced stress by high ambient temperatures, which could cause the defect generation, $\mathrm{Mg}-\mathrm{H}$ dissociation, contact degradation, and other effects $[15,16,17,18,19]$. These mechanisms might be useful to analyze the different aging behaviors of micro-LEDs, although the operation current densities are quite different. Furthermore, in micro-LED applications, a large micro-LED size (e.g. $60 \mu \mathrm{m})$ may be chosen to deliver high absolute light output power but a small size (e.g. $20 \mu \mathrm{m}$ ) may also be favored for achieving high current density and high modulation bandwidth $[1,9]$. In this case, it is important to study the size-dependent aging characteristics for choosing suitable micro-LED size in specific applications. For micro-LEDs with different sizes, the effects of thermal management and sidewall defects are strongly different $[9,10]$, so it is expected to observe strong size-dependent aging characteristics and the analyse of the size dependence will also help a deep understanding of the high-current-density aging behaviors.

In this work, we investigate the aging characteristics of micro-LEDs with different sizes at an extremely high current density $3.5 \mathrm{kA} / \mathrm{cm}^{2}$ for the first time, which is quite 
different from aging study on conventional broad-area LEDs. And this study provides directions to develop high-reliability LEDs (not limited to micro-LEDs) working at high current densities. The smaller micro-LEDs demonstrated less light output power degradation, which is a strong advantage for micro-LEDs. The high current density can help identify the effect of current-induced stress clearly, and the different selfheating effect of these micro-LEDs also leads to different thermal-induced stress. With much less thermal-induced stress, the smaller micro-LEDs demonstrate significantly better aging characteristics than larger micro-LEDs. In addition, the size dependence indicates the defects were mainly generated in the active region during aging test. Based on these results, high-power and high-reliability cluster LED devices composed of a number of micro-LEDs have been designed, fabricated and characterized. These cluster LED devices are able to work more reliable than normal broad-area LEDs, which is an important advantage for their applications in SSL and VLC.

\section{Experimental Details}

Top-emission blue InGaN micro-LED device was fabricated using a commercial wafer on $c$-plane sapphire substrates from SuperNova company. The wafer has a typical p-i-n structure with an $n$-GaN layer, an active region of InGaN/GaN multiple quantum wells (MQWs), an AlGaN layer, and a $p$-GaN layer. The design and fabrication techniques of the micro-LEDs were similar to those in our previous studies $[9,20]$. We started the micro-LED fabrication processes from depositing $\mathrm{Ni} / \mathrm{Au}(10 \mathrm{~nm} / 25 \mathrm{~nm})$ layers on the $p$ GaN surface, after which reactive-ion etching and inductively coupled plasma etching were employed to etch away the $\mathrm{Ni} / \mathrm{Au}$ and GaN-based epitaxial layers, respectively, to define micro-LED mesas. The samples were thermally annealed in purified air to form the Ni/Au current spreading layer. Then, a silicon dioxide layer was deposited by plasma-enhance chemical vapour deposition, and the apertures for each micro-LED mesa were opened by BOE wet etching. Finally, Ti/Au $(50 \mathrm{~nm} / 200 \mathrm{~nm})$ were deposited as the interconnection tracks and wire-bonding pads. The schematic structure of the microLEDs is shown in figure 1(a). The fabricated micro-LEDs have a emission wavelength $\sim 445 \mathrm{~nm}$ and the micro-LED efficiency is similar to that in [14]. Micro-LED sizes of $20 \mu \mathrm{m}, 40 \mu \mathrm{m}$ and $60 \mu \mathrm{m}$ in diameter were chosen for aging test at a current density $3.5 \mathrm{kA} / \mathrm{cm}^{2}$ under room temperature $\sim 25{ }^{\circ} \mathrm{C}$. These micro-LEDs were mounted on a $\mathrm{PCB}$ with a fixed distance to a Si detector for measurement of light output power and the ambient temperature was monitored by a thermometer. Both the micro-LED sizes and the selected test current density are typical for micro-LED applications $[1,3,7]$. Furthermore, at $3.5 \mathrm{kA} / \mathrm{cm}^{2}$, the three micro-LEDs will show significantly different selfheating effect [9], which helps clarify the effects of current-induced and thermal-induced stresses. Note that the self-heating effect at $3.5 \mathrm{kA} / \mathrm{cm}^{2}$ can lead to device failure for a larger micro-LED (e.g. $80 \mu \mathrm{m})$ [9]. To evaluate the self-heating effect, the junction temperatures of the micro-LEDs were obtained using a emission spectral shift method. We measured the temperature-dependent electroluminescence (EL) spetra under pulsed 

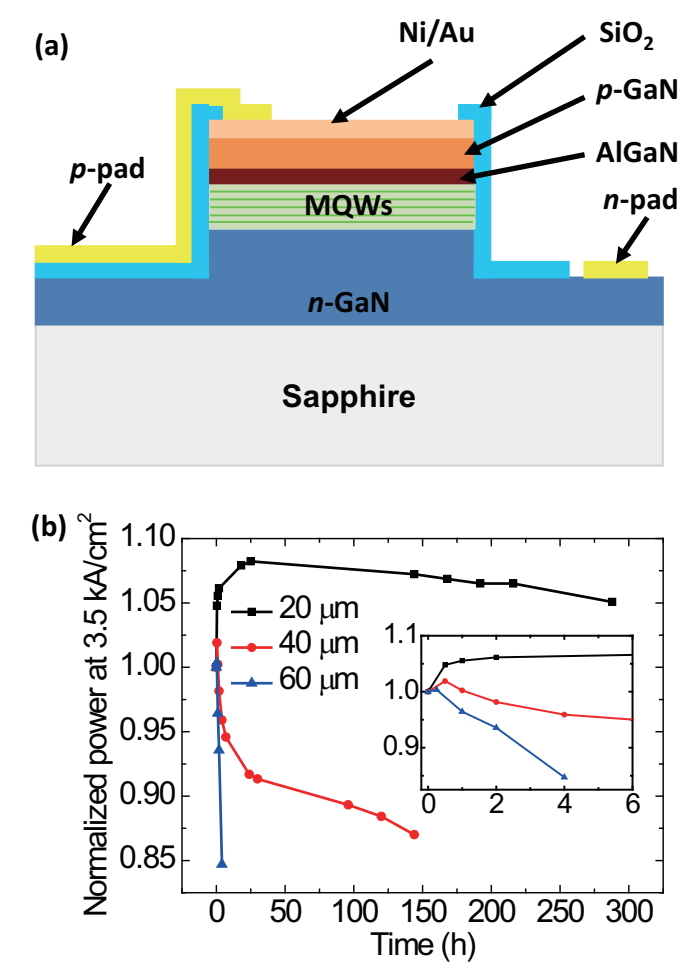

Figure 1. (a) Schematic structure of the fabricated micro-LEDs in this work. (b) Normalized light output power versus operation time of micro-LEDs with sizes of 20 $\mu \mathrm{m}, 40 \mu \mathrm{m}$ and $60 \mu \mathrm{m}$. The inset figure is to clearly show the data in the first $6 \mathrm{~h}$ period.

operation at $3.5 \mathrm{kA} / \mathrm{cm}^{2}$ to estimate the junction temperature of micro-LEDs under direct current during aging test $[9,21]$.

In addition, to adopt the micro-LED technology for applications in SSL and VLC, a cluster LED device was designed to achieve a high-power LED. The cluster LED consists of a $9 \times 9$ micro-LED array with flip-chip geometry. The gap distance between these micro-LEDs is $3 \mu \mathrm{m}$ and each micro-LED has a diameter of $30 \mu \mathrm{m}$ with fabrication processes similar to those in [22]. Another broad-area LED with size of $300 \mu \mathrm{m} \times 300$ $\mu \mathrm{m}$ was also fabricated for comparison.

\section{Results and discussion}

\subsection{Micro-LED aging characteristics}

In figure 1(b), the aging characteristics of three micro-LEDs show strong size dependence. Compared with the initial light output power at $0 \mathrm{~h}$, the power of the $20 \mu \mathrm{m}$ micro-LED increased $5 \%$ at $288 \mathrm{~h}$; the power of the $40 \mu \mathrm{m}$ micro-LED dropped $10 \%$ at $\sim 70 \mathrm{~h}$; the power of the $60 \mu \mathrm{m}$ micro-LED decreased $10 \%$ at $\sim 3 \mathrm{~h}$. For the 20 $\mu \mathrm{m}$ micro-LED, to achieve $10 \%$ power reduction, a linear mathematical fitting gives a value of operation time $\sim 1050 \mathrm{~h}[15,23]$. The purpose of the experiment is to compare the size-dependent aging characteristics, and from our experimental data the $20 \mu \mathrm{m}$ 
micro-LED already shows advantages over its counterparts. Note that only the $60 \mu \mathrm{m}$ micro-LED failed after the test, and randomly selected micro-LEDs with different sizes have been tested at different aging current densities to confirm the size-dependent trend in figure 1(b) is reliable. The light output power versus current $(L-I)$ curves of these micro-LEDs measured before and after aging are shown in figure 2. Except the $20 \mu \mathrm{m}$ micro-LED at high current densities, all other data indicate the light output power of the micro-LEDs degraded after aging. With longer aging time, we expect that the light output power of the $20 \mu \mathrm{m}$ micro-LED at high current densities would degrade eventually, as discussed in figure 1(b). Generally, the smaller micro-LEDs demonstrate significantly better aging characteristics over larger micro-LEDs at the same operation current density. Compared with the aging behaviors of broad-area LEDs at $\sim 100 \mathrm{~A} / \mathrm{cm}^{2}$ $[11,12,13]$, the small-size micro-LEDs, e.g. $20 \mu \mathrm{m}$ micro-LED, can be operated at an extremely higher operation current density $3.5 \mathrm{kA} / \mathrm{cm}^{2}$ with little degradation. We expect that smaller LEDs with size less than $20 \mu \mathrm{m}$ and even nano-LEDs can show better aging properties. The strong size effect on LED aging characteristics suggests a promising approach to improve the LED reliability.

Then, we analyze the underlying mechanisms of the aging characteristics in details. Through a close inspection in figure 1(b) and the inset figure, it is found that the light output powers of all the micro-LEDs increase initially for a certain period (denoted as the first stage) and then drop (denoted as the second stage). The increase periods are $\sim 25 \mathrm{~h}, \sim 0.5 \mathrm{~h}$ and $\sim 0.25 \mathrm{~h}$ for the $20 \mu \mathrm{m}, 40 \mu \mathrm{m}$ and $60 \mu \mathrm{m}$ micro-LEDs, respectively. Thus, the competition of two mechanisms on the micro-LED aging characteristics is expected, which will be analyzed in the following part.

The current versus voltage $(I-V)$ characteristics for the $20 \mu \mathrm{m}$ micro-LED are shown in figure 3(a) and (b) in linear scale and semi-logarithmic scale, respectively. The series resistance $\left(R_{\text {series }}\right)$ can be derived from the $I$ - $V$ characteristics using $R_{\text {series }}=$ $d V / d I$. We find that the $R_{\text {series }}$ at a high voltage (e.g. $4.5 \mathrm{~V}$ ) shows a fast reduction from $\sim 670 \Omega$ at $0 \mathrm{~h}$ to $\sim 400 \Omega$ at $0.5 \mathrm{~h}$ and then gradually decreases to $\sim 340 \Omega$ at 25 $\mathrm{h}$ for the $20 \mu \mathrm{m}$ micro-LED, which is synchronous with the fast and slow increase of its light output power at the first stage in figure 1(b). Similar phenomenon can be roughly seen for the $40 \mu \mathrm{m}$ and $60 \mu \mathrm{m}$ micro-LEDs, although their power increasing periods are comparably short. The $R_{\text {series }}$ at a high voltage is mainly dominated by the $p$-GaN and $p$-contact resistances [24]. It has been reported that at an elevated temperature $250{ }^{\circ} \mathrm{C}$ the $p$-contact resistance increased with longer operation time in [19]. For microLEDs with junction temperatures far below $250{ }^{\circ} \mathrm{C}$ as discussed later, self-annealing of the $p$-contact is not possible and therefore we assume that the $p$-contact resistance of micro-LEDs increased with longer aging time. In this case, the $R_{\text {series }}$ reduction in figure 3 was mainly dominated by the reduction of $p$-GaN resistance. For conventional broad-area LEDs, the power increase during aging test has also been observed and was attributed to the dissociation of $\mathrm{Mg}-\mathrm{H}$ complexes in $p$-GaN due to the effect of hot electrons $[16,25]$. Regarding our micro-LEDs, the operation current density 3.5 $\mathrm{kA} / \mathrm{cm}^{2}$ is much higher than that of broad-area LEDs, leading to higher probability 

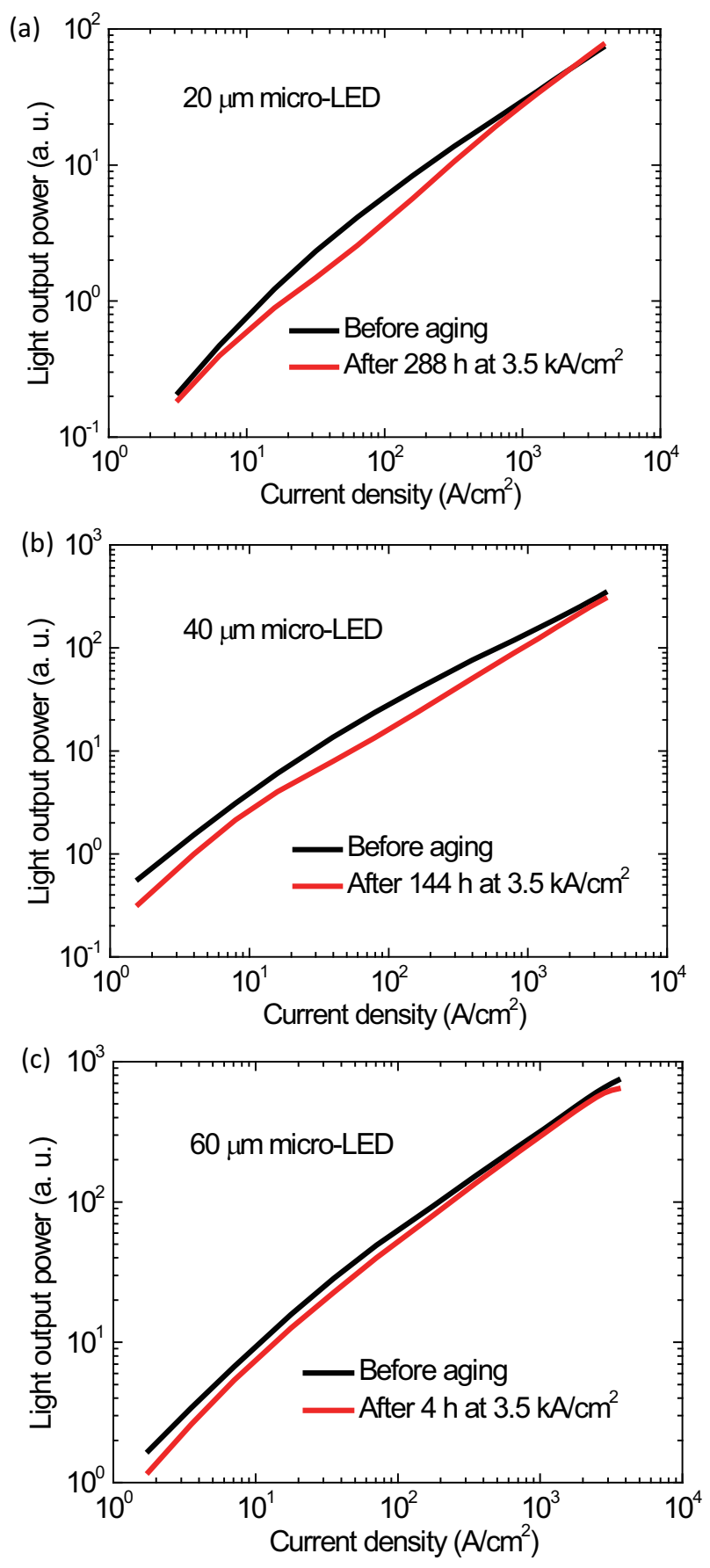

Figure 2. The $L-I$ curves measured before and after aging of micro-LEDs with sizes of (a) $20 \mu \mathrm{m}$, (b) $40 \mu \mathrm{m}$ and (c) $60 \mu \mathrm{m}$.

of $\mathrm{Mg}-\mathrm{H}$ complex dissociation. Thus, the injection current leads to the dissociation of Mg-H complex, $R_{\text {series }}$ reduction, and the power increase at the first operation stage.

We also analyze the $I-V$ characteristics to find the degradation mechanisms at the second stage, as shown in figure $4(\mathrm{a})$ and (b) for the $40 \mu \mathrm{m}$ and $60 \mu \mathrm{m}$ micro-LEDs, respectively. In the region from $1.5 \mathrm{~V}$ to $2 \mathrm{~V}$, for the $60 \mu \mathrm{m}$ micro-LED, tunnelling 

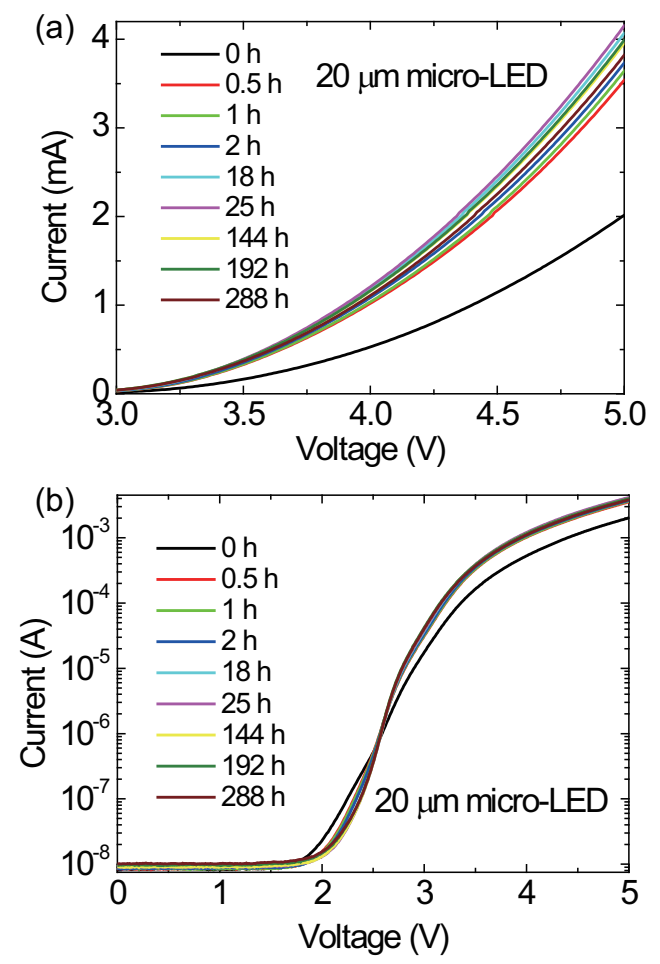

Figure 3. The $I$ - $V$ curves of a $20 \mu \mathrm{m}$ micro-LED at different operation times in (a) linear scale and (b) semi-logarithmic scale.

current increases significantly with longer operation time [26]. In comparison, a gradual increase of the tunnelling current can be found after $\sim 30 \mathrm{~h}$ for the $40 \mu \mathrm{m}$ micro-LED and almost no change is found in this region for the $20 \mu \mathrm{m}$ micro-LED (figure $3(\mathrm{~b})$ ). A large tunnelling current indicates more defects were generated during degradation [16, 27, 28]. In figure 2, the reduction of light output power after aging test, especially at low current densities, also indicates the increased defect-related non-radiation recombination and more defect generation $[19,29]$. Thus, the size dependence of $I-V$ characteristics indicates defects were generated faster in larger micro-LEDs, leading to faster power degradation (the second stage) and a shorter power increasing period (the first stage) in figure 1(b).

Regarding the defect generation during degradation, it was reported that the sidewall defect effects of an LED consisting of a micro-LED array were the key degradation mechanisms at a low operation current density of $\sim 5 \mathrm{~A} / \mathrm{cm}^{2}$ [23]. From this point, the smaller micro-LEDs might degrade more due to their higher ratio of sidewall area to the mesa area which contradicts our experimental results. Thus, our study indicates, for high-current-density degradation, the additional defects were mainly generated from the active region instead of the sidewalls of the micro-LEDs.

To explore the mechanisms of less light output power degradation of smaller microLEDs at the same operation current density, we estimate the self-heating effect of the three micro-LEDs by measuring their junction temperature $[9,21]$. At $3.5 \mathrm{kA} / \mathrm{cm}^{2}$ the 

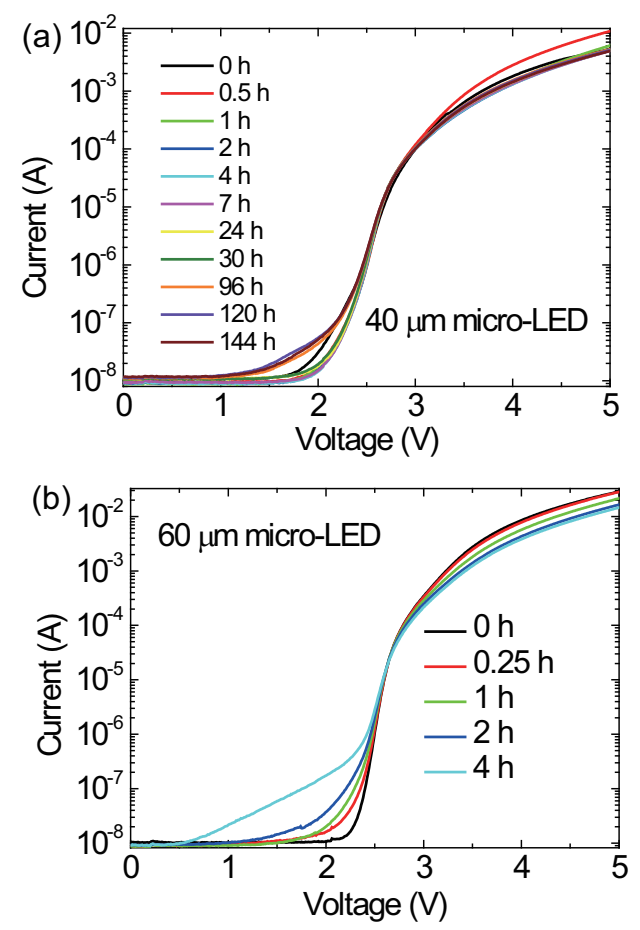

Figure 4. The $I$ - $V$ characteristics of (a) a $40 \mu \mathrm{m}$ micro-LED and (b) a $60 \mu \mathrm{m}$ microLED in semi-logarithmic scale at different operation times.

junction temperatures of the $20 \mu \mathrm{m}, 40 \mu \mathrm{m}$ and $60 \mu \mathrm{m}$ micro-LEDs were estimated to be $\sim 5{ }^{\circ} \mathrm{C}, \sim 16{ }^{\circ} \mathrm{C}$ and $\sim 40{ }^{\circ} \mathrm{C}$ higher than room temperature, respectively. The junction temperature of the $20 \mu \mathrm{m}$ micro-LED can be approximately considered as similar to the room temperature, due to its excellent current spreading and heat dissipation effects $[9,10,30]$. It was reported that a higher ambient temperature led to more power degradation by several studies $[17,18,19]$, and we assume this temperature effect also applies to the micro-LEDs in this work. The degradation mechanisms in the active region may be the thermally activated defect generation [17], and other possible effects. Based on these analyses, we can clarify the effects of current-induced stress by high injection current density and thermal-induced stress by additional high junction temperature: at room temperature without additional junction temperature, high-current-densityinduced stress causes little degradation of light output power (e.g. $20 \mu \mathrm{m}$ micro-LED); the combination of the effects of high-junction-temperature and high-current-densityinduced stresses leads to large power degradation (e.g. $40 \mu \mathrm{m}$ and $60 \mu \mathrm{m}$ micro-LEDs).

\subsection{High-power cluster LEDs consisting of a micro-LED array}

The VLC and SSL systems are expected to be combined together for indoor applications, so developing LEDs with both high modulation bandwidth and high light output power is necessary. The micro-LEDs have advantages to achieve high current density, high density of light output power and high modulation bandwidth, but the total light output 

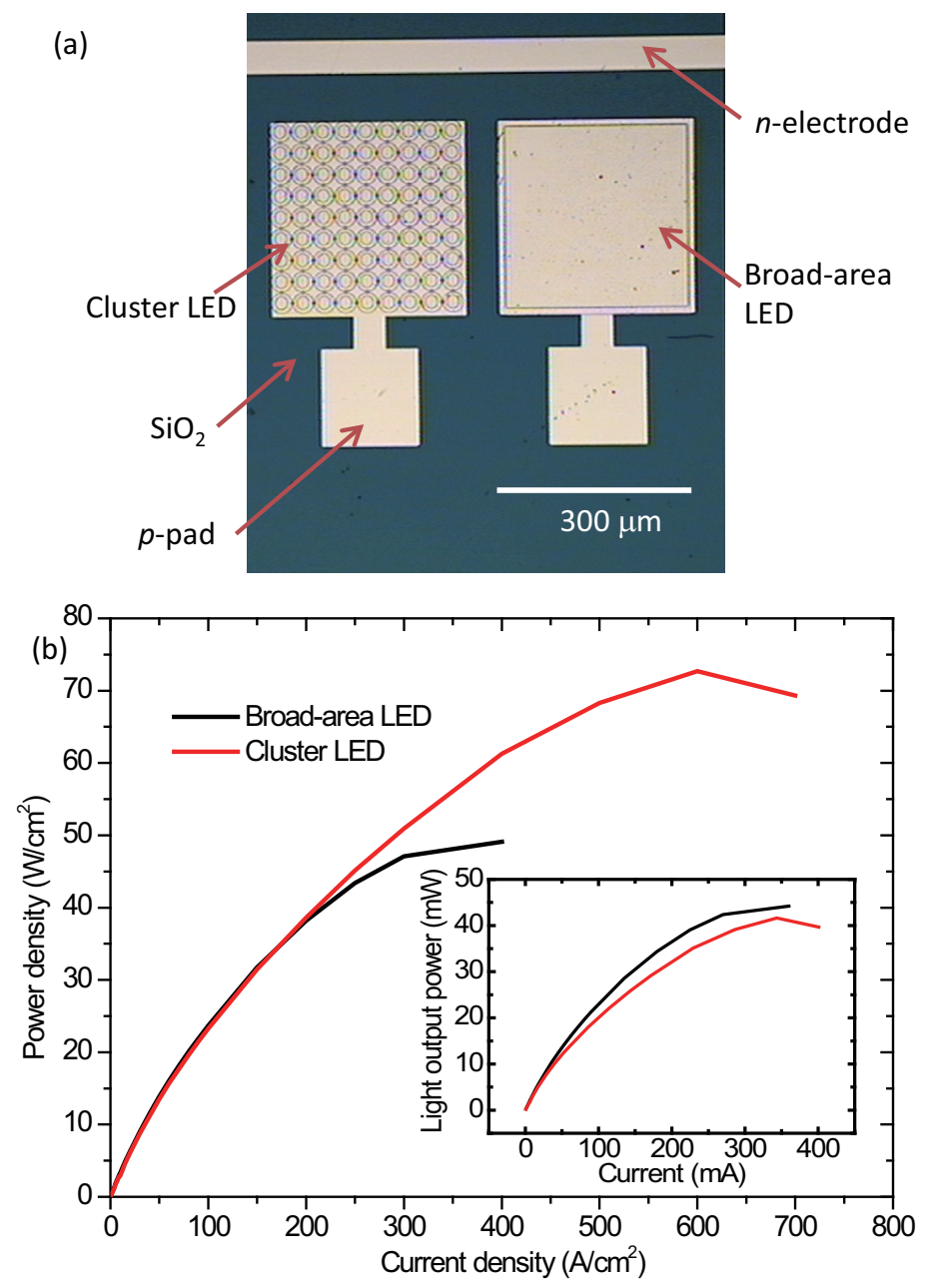

Figure 5. (a) Microscopic image of cluster LEDs and broad-area LEDs. (b) Light output power density versus current density and light output power versus current (inset) of cluster LEDs and broad-area LEDs. The size of the broad-area LED is $300 \mu \mathrm{m} \times 300 \mu \mathrm{m}$. The cluster LED consists of a $9 \times 9$ micro-LED array with each micro-LED $30 \mu \mathrm{m}$ in diameter and gap distance $3 \mu \mathrm{m}$ between the micro-LEDs.

power from a single micro-LED is pretty low due to the small emission area $[1,9]$. In addition, high light output power also helps increase the data transmission speed of VLC [20]. Here, the micro-LED technology and proposed mechanisms can be effectively adopted to design cluster LEDs with comprised high-current-density, high-power and high-reliability for applications in both VLC and SSL.

The microscopic image of the cluster LED and conventional broad-area LED is shown in figure 5(a). The cluster device has a filling factor of $\sim 0.64$ in an area of 300 $\mu \mathrm{m} \times 300 \mu \mathrm{m}$, compared with the broad-area LED. Figure 5(b) and the inset figure show the characteristics of light output power density versus current density and the light output power versus current measured by an integrating sphere system, respectively. The light output power of the cluster LED is only $~ 10 \%$ lower than the broad-area LED at the same current, although the light emission area is reduced by $\sim 36 \%$. We attribute 

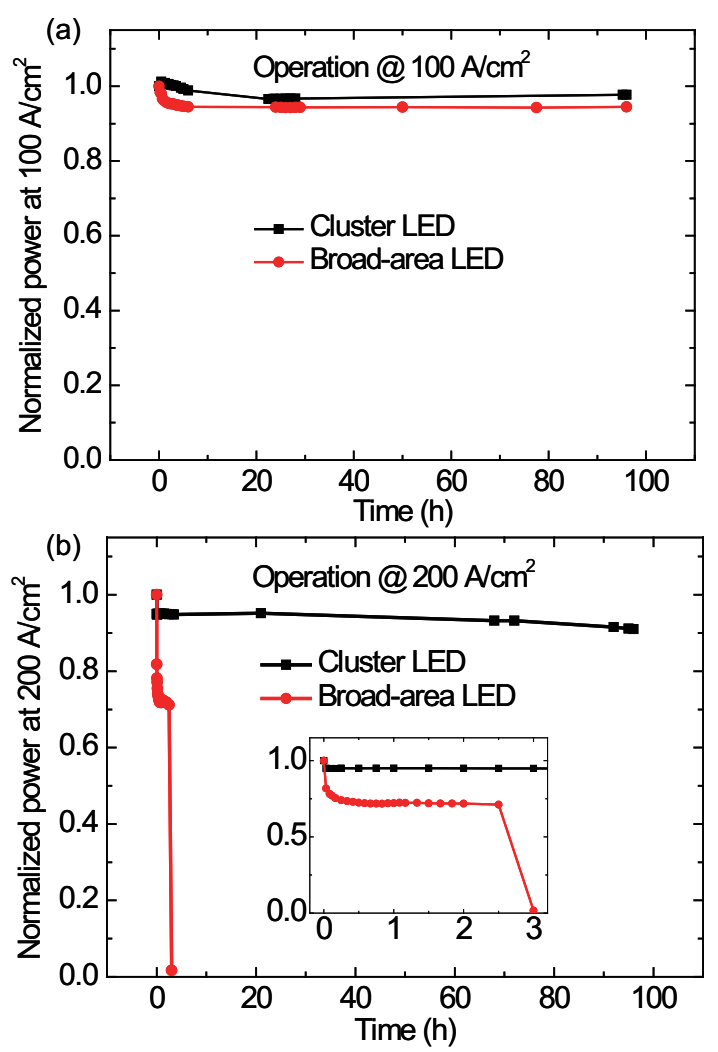

Figure 6. Normalized light output power versus operation time of cluster LEDs and broad-area LEDs $(300 \mu \mathrm{m} \times 300 \mu \mathrm{m})$ at operation current densities of (a) $100 \mathrm{~A} / \mathrm{cm}^{2}$ and (b) $200 \mathrm{~A} / \mathrm{cm}^{2}$ (inset: degradation in $3 \mathrm{~h}$ period).

the phenomenon to the higher driven current density due to the smaller emission area, the excellent current spreading and thermal dissipation effects of cluster LEDs [9, 10, 30]. The broad-area LED has a thermal saturation current between $400 \mathrm{~A} / \mathrm{cm}^{2}$ and 500 $\mathrm{A} / \mathrm{cm}^{2}$, as the device failed when increasing the current density to $500 \mathrm{~A} / \mathrm{cm}^{2}$ during the experimental test. In comparison, the cluster LED has a higher thermal saturation current density of $\sim 600 \mathrm{~A} / \mathrm{cm}^{2}$. It was reported that a higher current density could lead to a higher modulation bandwidth [1], so the cluster device can be driven faster than the broad-area LED. Also, the higher thermal saturation current density indicates that the thermal effect of the cluster LED is relatively weaker than that of the broad-area LED and thereby better aging characteristics are expected for the cluster LED at the same current density.

At the same operation current density $100 \mathrm{~A} / \mathrm{cm}^{2}$ as shown in figure $6(\mathrm{a})$, at $96 \mathrm{~h}$ the power of the cluster LED dropped $\sim 2.3 \%$ while the power of the broad-area LED dropped $\sim 5.5 \%$, indicating slightly better aging characteristics of the cluster LED. In comparison, at $200 \mathrm{~A} / \mathrm{cm}^{2}$ as shown in figure 6(b), the power of the cluster LED dropped $\sim 9 \%$ at $96 \mathrm{~h}$ while the power of the broad-area LED decreased $\sim 29 \%$ at $2.5 \mathrm{~h}$ and suffered catastrophic power reduction at $3 \mathrm{~h}$, demonstrating significant advantage of the 
cluster LED. The advantage can be caused by the improved current spreading, reduced self-heating and better thermal dissipation effects of the cluster LED [9, 10, 30]. We have noticed that, different from micro-LED aging characteristics in figure $1(\mathrm{~b})$, an initial increase of light output power was not found in figure 6 , which also can be explained by the mechanisms mentioned above. On the one hand, the operation current density of large-area LEDs is much smaller than micro-LEDs, so we expect a slower power increase due to the hot electron effect on $\mathrm{Mg}-\mathrm{H}$ dissociation; on the other hand, due to the general larger current crowding and self-heating effects of these large-area LEDs than micro-LEDs $[9,30]$, the thermal effect caused very fast power degradation, and even the power increase stage can not be seen. Consequently, the micro-LED technique is a promising approach to design high-reliability and high-power LEDs for applications in SSL and VLC, especially at high current densities.

\section{Conclusion}

In summary, the aging characteristics of micro-LEDs at $3.5 \mathrm{kA} / \mathrm{cm}^{2}$ have been systematically studied including the size dependence and mechanisms. Micro-LEDs with smaller sizes demonstrated better aging characteristics, suggesting an apporoach to improve the LED reliability. The initial increase and latter decrease of light output power have been attributed to the competition of $\mathrm{Mg}-\mathrm{H}$ dissociation and defect generation. The size dependence proves that the additional defects were mainly generated in the active region of micro-LEDs. The high operation current density 3.5 $\mathrm{kA} / \mathrm{cm}^{2}$ causes little degradation of the $20 \mu \mathrm{m}$ micro-LEDs, proving that only high current density at room temperature is not the main cause of degradation; however, strong degradation of larger micro-LEDs, e.g. $60 \mu \mathrm{m}$ micro-LEDs, demonstrates the addition of junction temperature on the micro-LEDs plays a significant role on the microLED degradation. Furthermore, the micro-LED technology has been used to develop cluster LEDs with both high light output power and high reliability for applications in SSL and VLC.

\section{Acknowledgments}

This work was supported by the Engineering and Physical Sciences Research Council under Grant EP/K00042X/1 and by the Start-up Research Funding JJH1232106 from Fudan University.

\section{References}

[1] McKendry J J D, Massoubre D, Zhang S, Rae B R, Green R P, Gu E, Henderson R K, Kelly A E and Dawson M D 2012 J. Lightwave Technol. 3061

[2] Li X, Bamiedakis N, Wei J L, McKendry J J D, Xie E, Ferreira R, Gu E, Dawson M D, Penty R V and White I H 2015 J. Lightwave Technol. 333571 
[3] Zhang H X, Massoubre D, McKendry J, Gong Z, Guilhabert B, Griffin C, Gu E, Jessop P E, Girkin J M and Dawson M D 2008 Opt. Express 169918

[4] Herrnsdorf J, Wang Y, McKendry J J D, Gong Z, Massoubre D, Guilhabert B, Tsiminis G, Turnbull G A, Samuel I D W, Laurand N et al. 2013 Laser Photonics Rev. 71065

[5] Poher V, Grossman N, Kennedy G T, Nikolic K, Zhang H X, Gong Z, Drakakis E M, Gu E, Dawson M D, French P M W et al. 2008 J. Phys. D: Appl. Phys. 41094014

[6] Day J, Li J, Lie D Y C, Bradford C, Lin J Y and Jiang H X 2011 Appl. Phys. Lett. 99031116

[7] Guilhabert B, Massoubre D, Richardson E, McKendry J J D, Henderson R K, Watson I M, Gu E and Dawson M D 2012 IEEE Photonics Technol. Lett. 242221

[8] Fan Z Y, Lin J Y and Jiang H X 2008 J. Phys. D: Appl. Phys. 41094001

[9] Gong Z, Jin S, Chen Y, McKendry J, Massoubre D, Watson I M, Gu E and Dawson M D $2010 \mathrm{~J}$. Appl. Phys. 107013103

[10] Tian P, McKendry J J D, Gong Z, Guilhabert B, Watson I M, Gu E, Chen Z, Zhang G and Dawson M D 2012 Appl. Phys. Lett. 101231110

[11] Hahn B, Galler B and Engl K 2014 Jpn. J. Appl. Phys. 53100208

[12] Meneghini M, Tazzoli A, Meneghesso G and Zanoni E 2010 IEEE T. Electron Dev. 57108

[13] Meneghini M, Lago M D, Trivellin N, Meneghesso G and Zanoni E 2014 IEEE T. Ind. Appl. 50 78

[14] Tian P, McKendry J J D, Herrnsdorf J, Watson S, Ferreira R, Watson I M, Gu E, Kelly A E and Dawson M D 2014 Appl. Phys. Lett. 105171107

[15] Chang M H, Das D, Varde P V and Pecht M 2012 Microelectron. Reliab. 52762

[16] Moe C G, Reed M L, Garrett G A, Sampath A V, Alexander T, Shen H, Wraback M, Bilenko Y, Shatalov M, Yang J et al. 2010 Appl. Phys. Lett. 96213512

[17] Jung E, Kim M S and Kim H 2013 IEEE T. Electron Dev. 60186

[18] Gong Z, Gaevski M, Adivarahan V, Sun W, Shatalov M and Asif Khan M 2006 Appl. Phys. Lett. 88121106

[19] Meneghini M, Trevisanello L R, Meneghesso G and Zanoni E 2008 IEEE T. Device Mat. Re. 8 323

[20] Tian P, McKendry J J D, Gong Z, Zhang S, Watson S, Zhu D, Watson I M, Gu E, Kelly A E, Humphreys C J et al. 2014 J. Appl. Phys. 115033112

[21] Lin Y, Gao Y L, Lu Y J, Zhu L H, Zhang Y and Chen Z 2012 Appl. Phys. Lett. 100202108

[22] Yang W, Zhang S, McKendry J J D, Herrnsdorf J, Tian P, Gong Z, Ji Q, Watson I M, Gu E, Dawson M D et al. 2014 J. Appl. Phys. 116044512

[23] Li Z L, Li K H and Choi H W 2010 J. Appl. Phys. 108114511

[24] Guo X and Schubert E F 2001 J. Appl. Phys. 904191

[25] Liu L, Ling M, Yang J, Xiong W, Jia W and Wang G 2012 J. Appl. Phys. 111093110

[26] Cao X A, Stokes E B, Sandvik P M, LeBoeuf S F, Kretchmer J and Walker D 2002 IEEE Electr. Device L. 23535

[27] Cao X, Sandvik P M, LeBoeuf S F and Arthur S D 2003 Microelectron. Reliab. 431987

[28] Polyakov A Y, Smirnov N B, Govorkov A V, Kim J, Luo B, Mehandru R, Ren F, Lee K P, Pearton S J, Osinsky A V and E N P 2002 J. Appl. Phys. 915203

[29] Shao X, Lu H, Chen D, Xie Z, Zhang R and Zheng Y 2009 Appl. Phys. Lett. 95163504

[30] Ploch N L, Rodriguez H, Stolmacker C, Hoppe M, Lapeyrade M, Stellmach J, Mehnke F, Wernicke T, Knauer A, Kueller V et al. 2013 IEEE T. Electron Dev. 60782 